\title{
Mobility Force Model for Microscopic Simulation of Motorcycle Traffic Behavior
}

\author{
Tienpen $\mathrm{Hsu}^{*}$ \\ Institute of Civil Engineering \\ National Taiwan University \\ Taipei, Taiwan
}

\author{
Yuchen Chen \\ Institute of Civil Engineering \\ National Taiwan University \\ Taipei, Taiwan
}

\begin{abstract}
This paper presents the conceptual structure of a novel microscopic traffic flow behavior simulation model for motorcycle traffic based on a mobility force structure, called mobility force car-following model. The mobility force is defined as the interactive force of motorcycle by surrounding vehicles and environment of lane layout. The kinetic behavior of the motorcycle will be induced and constrained by the force created due to the spaces surrounding. The concept is to describe the driving behavior using a series of forces of the attraction and repulsion related to the vehicle and spaces surrounding in front of the motorcycle. The model can describe both of the lateral motion and longitudinal motion in views to the motorcycle driving feature. Through a series of calibration for the parameters of the model, the regression statistical significance and the validation of the model output using the field survey data, the results illustrated the enough accuracy of the result of model output. The model is then verified to be suitable to describe the real motorcycle driving behavior preliminarily.
\end{abstract}

Keywords-car-following model; mobility force; force field; microscopic simulation; motorcycle

\section{INTRODUCTION}

Traditional car-following model assumed vehicles only move along a line within the lane following to the front vehicle, while not doing lane change. The car following models explained the motion of vehicles basically only in one dimension. The vehicle will react to the front vehicle under the stimulus by front vehicle basing on the distance and relative speed etc, such as in the GM car-following model. However, for the traffic with high composition of motorcycles, the driving behavior responses to the possible space in front of the vehicles. The vehicle driving behavior, especially regarding the motorcycle, will be influenced not only by the front car, but also by the car surrounding it nearby, and nevertheless by the width of lane and the lateral clearance. Therefore, this paper is newly to create a novel car-following model under consideration of all the spaces both longitudinal and lateral surrounding the motorcycle. The idea originally comes from the concept of force field interactive between the space and vehicles. Therefore, it is named as Mobility Force Model.

\section{CONCEPTURAL STRUCTURE OF MOBILITy ForCE MODEL}

\section{A. Basic Concept}

In the past, Hsu etc., had proposed a car-following behavior model based on the concept of Newton's law of universal gravitation, called "gravitational car-following model" [1]. Under the assumption of car driving behavior of pursuing the fast speed as possible to achieve desired speed, the vehicle will always want to move to the space where no vehicles occupied, and it avoids collision with other vehicles. Therefore, the surrounding not occupied space in front of the car will create an attraction force to push the vehicle move to that position as soon as possible. The attraction force concept is then generated. Though the calibration of the parameters using field survey data, the accuracy is not acceptable with respects of statistics significance. However, the force field concept could be a possible direction for developing advanced car-following model. On the other hand, Helbing created a social force model to describe the pedestrian walking behavior [2]. It takes the pedestrian walking behavior as the result of the interactive force between the pedestrian based on the territory impact on the force to move the pedestrian. The social force model becomes then the practically successful model using in microscopic simulation software VSSIM. In this paper, the motorcycle driving behavior could be seen as similar reaction of force impact, but motorcycle has the mobility and should be constrained by acceleration and deceleration ability of motorcycle and safety concern of motorcyclist. Therefore, referring to the social force model and the force field interactive concept, a Mobility Force Model is then proposed at this research. If there is a vehicle which impedes the vehicle to move ahead under the limit of the distance, it will cause a repulsive force to stop the vehicle or reduce its speed accordingly. The vehicle will drive with the resulted acceleration affected by the force. However, there are a number of forces which influence the acceleration or deceleration at the same time. The driving behavior will be affected by the total force. The forces are generated by the space in front the vehicle or next to the vehicle. Therefore, at least, it supposes there are at least four forces affecting driving, as illustrated in Figure 1, which are:

1. Desired speed attraction force to move forwards, such as the difference between desired speed and present speed, $\mathrm{F}_{\mathrm{d}}$;

2. Interaction force by other vehicles surrounding nearby $F_{i}$, including which will obstruct following vehicle to move forwards, $F_{f}$ and attraction force generated by of lateral space from the car by the next, $F_{n}$;

Repulsive force by the lane marking for lane keeping, $F_{1}$. 


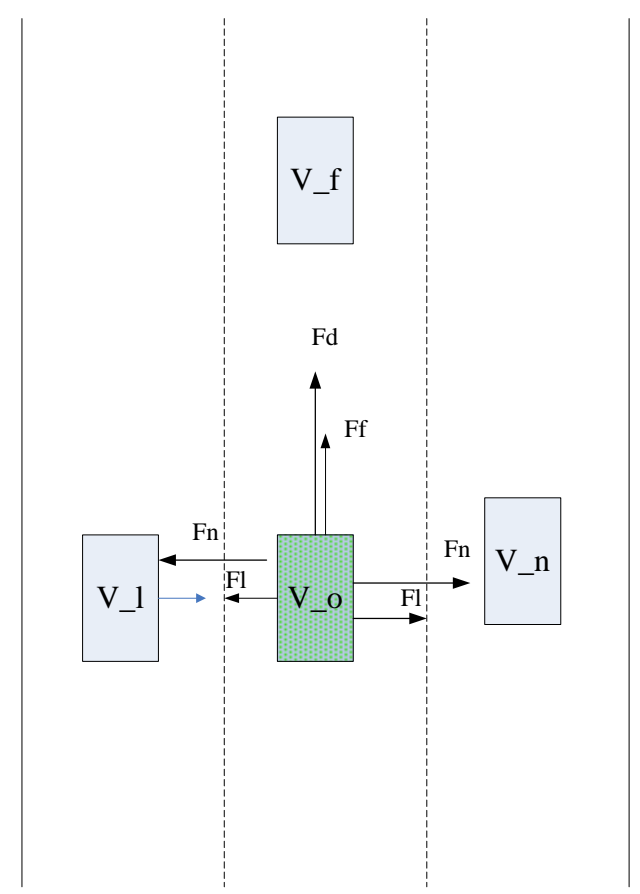

FIGURE I. FORCES OF DRIVING FORCE FIELD

\section{B. Desired Speed Force}

The desired speed attraction force $F_{d}$ is a longitudinal force caused by the attraction force for vehicle to achieve the desired speed. The force equation of $F_{d}$ is as follows.

$$
\vec{f}_{\alpha}^{d}(t)=\frac{1}{\tau_{\alpha}}\left(v_{\alpha}^{d}(t) \vec{e}_{\alpha}-\vec{v}_{\alpha}(t)\right)
$$

where $\vec{f}_{\alpha}^{0}(t)$ is desired speed force of the driver $\alpha$ influenced by desired speed $v_{\alpha}^{d}(t)$ and the expected driving direction $\vec{e}_{\alpha}$, spot speed $\vec{v}_{\alpha}(t)$ and reaction time $\tau_{\alpha}$.

\section{Interaction Force}

The strength of the interaction force Fi will be influenced by the distance and the driver vision angle change. The interaction force Fi can be denoted as combination of two parts as following equation.

$$
\vec{f}_{\alpha \beta}(t)=w\left(\theta_{\alpha \beta}\right) \vec{g}\left(\vec{r}_{\alpha \beta}(t)\right)
$$

where the $\theta_{\alpha \beta}$ is the angle between in front vehicle $\beta$ and the observed vehicle driver $\alpha$, and the $\vec{r}_{\alpha \beta}(t)$ is the relative position of $\alpha$ and $\beta$ at t time point.

$$
\vec{r}_{\alpha \beta}(t)=\vec{r}_{\alpha}(t)-\vec{r}_{\beta}(t)
$$

where the $\vec{r}_{\alpha}(t) 、 \vec{r}_{\beta}(t)$ are the position dimension of $\alpha$ and $\beta$, as shown in following Figure 2.

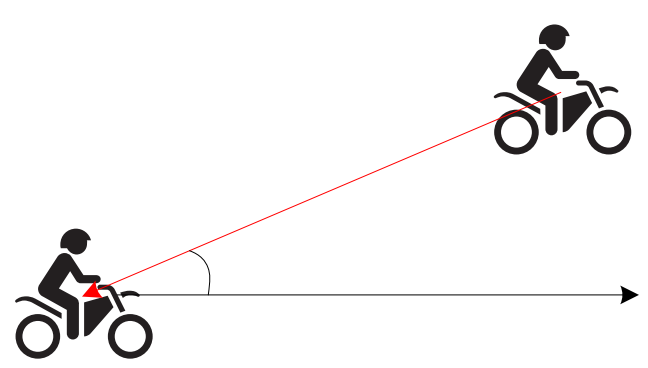

FIGURE II. THE RELATIVE POSITION OF THE VEHICLES

The $w\left(\theta_{\alpha \beta}\right)$ is the angle weight to denote the impact of the front vehicle on the driver. The interaction force is inversed with the distance, and denoted as $\vec{g}\left(\vec{r}_{\alpha \beta}(t)\right)$.

$$
\vec{g}\left(\vec{r}_{\alpha \beta}(t)\right)=-\nabla_{\vec{r}_{\alpha \beta}} V_{\alpha \beta}\left(\vec{r}_{\alpha \beta}(t)\right)
$$

where $V_{\alpha \beta}$ is the interaction force field. The force field is described as an ellipse shape field as illustrated in Figure 3. When the speed difference $d v$ between these two vehicles is zero, where exits a basic force field as shown in Figure 3.a, otherwise there is the relative force field as shown in Figure 3.b.
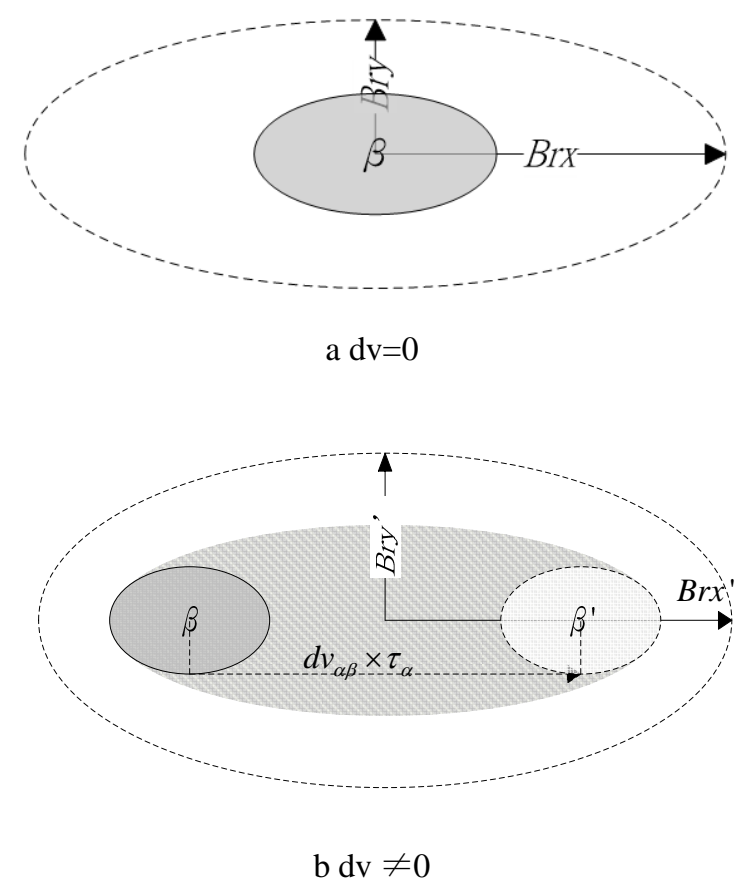

FIGURE III. INTERACTION FORCE FIELD

Combining with the speed difference and the distance into the ellipse force field dimension, the interaction force Fi is then as following equation. Its parameters requested being calibrated are listed in Table 1. 


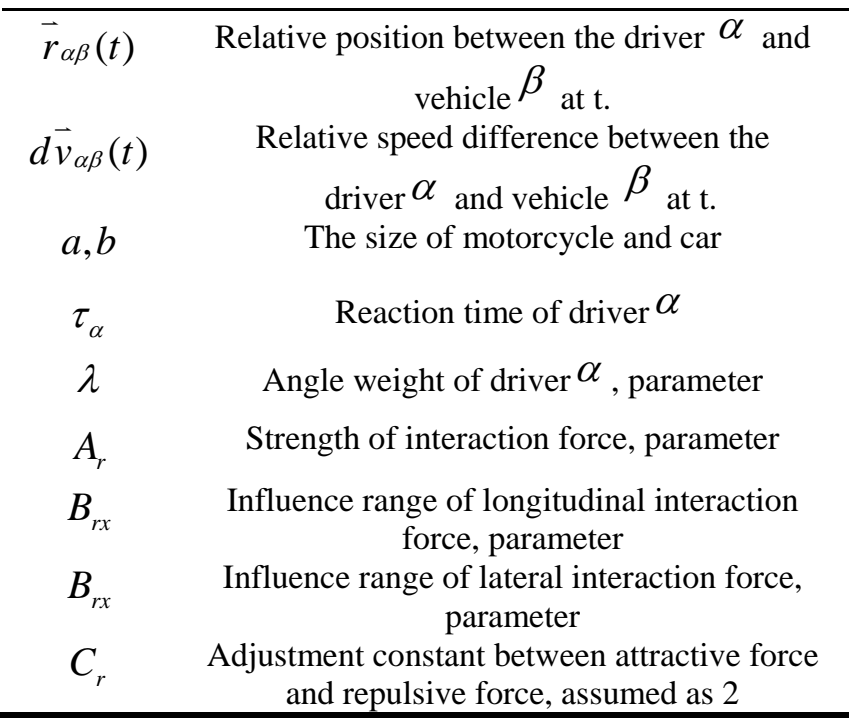

\section{Curb Lane Marking Force}

For the vehicle to drive on the curb lane, it should be constrained by the space closing to the lane marking for keeping safety side clearance. The force by curb lane marking $\mathrm{F}_{1}$ is illustrated in Figure 4.

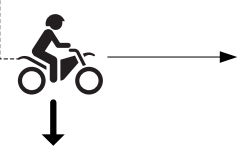

FIGURE IV. FORCE BY CURB LANE MARKING

$$
\vec{f}_{\alpha B}\left(\vec{r}_{\alpha B}(t)\right)=-\nabla_{\vec{r}_{\alpha B}} U_{B}\left(\left|\vec{r}_{\alpha}-\vec{r}_{B}\right|\right)
$$

where the distance to the curb is $\vec{r}_{\alpha B}=\vec{r}_{\alpha}-\vec{r}_{B}, \vec{r}_{\alpha}$ is the position of driver $\alpha$ and $\vec{r}_{B}$ is the position of curb lane marking, $U_{B}$ is the force field.

$$
U_{B}\left(\vec{r}_{\alpha B}(t)\right)=A_{U} e^{-\left|\vec{r}_{\alpha B}(t)\right| / B_{U}} \cdot \frac{\vec{r}_{\alpha B}(t)}{\left|\vec{r}_{\alpha B}(t)\right|}
$$

The parameters needed to be calibrated are listed in Table 2 .

Table 2 the Parameters of lane marking force.

$\begin{array}{cc}\vec{r}_{\alpha B}(t) & \begin{array}{c}\text { Relative position of driver } \alpha \text { to the lane } \\ \text { marking at time t. }\end{array} \\ A_{U} & \begin{array}{c}\text { Strength of the lane marking force, } \\ \text { parameter }\end{array} \\ B_{U} & \text { Range of the lane marking force field, } \\ \text { parameter }\end{array}$

\section{CALIBRATION OF MODEL PARAMETERS}

Using the maximal likelihood method, the parameters of the model are calibrated by referring the method addressed by Hoogendoorn [3].

\section{A. Maximal Likelihood Function}

The model with the random error item can be denoted as following equation.

$$
\vec{a}_{\alpha}(t+\tau)=\vec{f}_{\alpha}\left(\vec{v}_{\alpha}(t), \vec{r}_{\alpha}(t)-\vec{r}_{\beta}(t), \ldots \mid \theta_{\alpha}\right)+\vec{\varepsilon}_{\alpha}
$$

where $\vec{a}_{\alpha}$ is the acceleration resulted from the total force, $\vec{f}_{\alpha}$ is the model content of force and $\theta_{\alpha}$ denotes the set of all parameters, the $\vec{\varepsilon}_{\alpha}$ is the random error item which is assumed to follow normal distribution with mean 0 and standard deviation of . Through a field video survey, the data of position, relative distance and speed difference would be taken to calibrate the parameter set $\theta_{\alpha}$.

$$
\begin{gathered}
L_{k}\left(\theta_{\alpha}, \sigma_{\alpha}\right)=\frac{1}{\sigma_{\alpha} \sqrt{2 \pi}} e^{-\frac{\left(\vec{a}_{\alpha}^{o b s}\left(t_{k}+\tau\right)-\vec{a}_{\alpha}\left(t_{k}+\tau\right)\right)^{2}}{2 \sigma_{\alpha}^{2}}} \\
\vec{a}_{\alpha}\left(t_{k}+\tau \mid \theta_{\alpha}\right)=\vec{f}_{\alpha}\left(\vec{v}_{\alpha}^{o b s}(t), \vec{r}_{\alpha}^{o b s}(t)-\vec{r}_{\beta}^{o b s}(t), \ldots \mid \theta_{\alpha}\right)
\end{gathered}
$$

The likelihood function $L_{k}$ of each time step of traffic flow is as equation (8), where the $\vec{a}_{\alpha}^{o b s}\left(t_{k}+\tau\right)$ is the real observed acceleration rate, $\vec{a}_{\alpha}\left(t_{k}+\tau\right)$ is the model estimated acceleration rate as equation (9), which is dependent on the value of parameter $\theta_{\alpha}$. Using the data of $\mathrm{n}$ time steps, the likelihood estimator is as follows:

$$
\begin{aligned}
& L=L\left(\theta_{\alpha}, \sigma_{\alpha}\right)=\prod_{k=1}^{n} L_{k}\left(\theta_{\alpha}, \sigma_{\alpha}\right) \\
& \ln L\left(\theta_{\alpha}, \sigma_{\alpha}\right)=-\frac{n}{2} \ln \left(2 \pi \sigma_{\alpha}^{2}\right)-\frac{2}{2 \sigma_{\alpha}^{2}} \sum_{k=1}^{n}\left(\vec{a}_{\alpha}^{o b s}\left(t_{k}+\tau\right)-\vec{a}_{\alpha}\left(t_{k}+\tau \mid \theta_{\alpha}\right)\right)^{2} \\
& \frac{\partial \ln L}{\partial \sigma_{\alpha}^{2}}=0 \Rightarrow \hat{\sigma}_{\alpha}^{2}=\frac{1}{n} \sum_{k=1}^{n}\left(\vec{a}_{\alpha}^{o b s}\left(t_{k}+\tau\right)-\vec{a}_{\alpha}\left(t_{k}+\tau \mid \theta_{\alpha}\right)\right)^{2} \\
& \ln L\left(\theta_{\alpha}, \hat{\sigma}_{\alpha}\right)=-\frac{n}{2} \ln \left(\frac{2 \pi}{n} \sum_{k=1}^{n}\left(\vec{a}_{\alpha}^{o b s}\left(t_{k}+\tau\right)-\vec{a}_{\alpha}\left(t_{k}+\tau \mid \theta_{\alpha}\right)\right)^{2}\right)-\frac{n}{2} \\
& \hat{\theta}_{\alpha}=\arg \max \ln L\left(\theta_{\alpha}, \hat{\sigma}_{\alpha}\right)
\end{aligned}
$$

\section{B. Results of Parameter Calibration}

Using a video survey and image processing technique, the field observed traffic flow microscopic data are used to calibrate the parameter of the model. Table 3 lists the input variable values for modelling. The values come out through the field survey. Table 4 illustrates all the input constant and calibrated parameters 
TABLE III. INPUT VARIABLE VALUE OF THE MODEL

\begin{tabular}{|c|c|c|}
\hline \multicolumn{3}{|c|}{ Driver parameter } \\
\hline$\mu\left(v_{\max }^{d}\right)$ & $\begin{array}{l}\text { Mean for Maximal desired } \\
\text { speed distribution }\end{array}$ & $14.96 \mathrm{~m} / \mathrm{s}$ \\
\hline$\sigma\left(v_{\max }^{d}\right)$ & $\begin{array}{l}\text { Standard deviation for } \\
\text { Maximal desired speed } \\
\text { distribution }\end{array}$ & $3.55 \mathrm{~m} / \mathrm{s}$ \\
\hline$\tau$ & Reaction time & $1 \mathrm{~s}$ \\
\hline$C_{r}$ & $\begin{array}{l}\text { Adjustment constant between } \\
\text { attractive force and repulsive } \\
\text { force }\end{array}$ & 2 \\
\hline \multicolumn{3}{|c|}{ Size parameter of vehicle } \\
\hline$a_{m}$ & Size parameter of motorcycle & $1.2 \mathrm{~m}$ \\
\hline$b_{m}$ & Size parameter of motorcycle & $0.5 \mathrm{~m}$ \\
\hline$a_{c}$ & Size parameter of car & $2.5 \mathrm{~m}$ \\
\hline$b_{c}$ & Size parameter of car & $1.9 \mathrm{~m}$ \\
\hline
\end{tabular}

TABLE IV. CALIBRATION RESULTS OF PARAMETERS

\begin{tabular}{|c|c|c|}
\hline \multicolumn{3}{|c|}{ Desired speed force } \\
\hline$C$ & Constant of initial desired speed & 1.77 \\
\hline \multicolumn{3}{|c|}{ Lane marking force } \\
\hline$A_{U}$ & $\begin{array}{c}\text { Strength of the lane marking force, } \\
\text { parameter }\end{array}$ & $1.87 \mathrm{~m} / \mathrm{s} 2$ \\
\hline$B_{U}$ & $\begin{array}{c}\text { Range of the lane marking force } \\
\text { field, parameter }\end{array}$ & $1.80 \mathrm{~m}$ \\
\hline \multicolumn{3}{|c|}{ Interaction force } \\
\hline$\lambda$ & Angle weight of driver $\alpha$ & 0.11 \\
\hline$A_{r m}$ & $\begin{array}{l}\text { Strength of interaction force of } \\
\text { motorcycle }\end{array}$ & $0.63 \mathrm{~m} / \mathrm{s} 2$ \\
\hline$B_{r m x}$ & $\begin{array}{l}\text { Influence range of longitudinal } \\
\text { interaction force of motorcycle }\end{array}$ & $2.11 \mathrm{~m}$ \\
\hline$B_{r m y}$ & $\begin{array}{c}\text { Influence range of lateral } \\
\text { interaction force of motorcycle }\end{array}$ & $1.37 \mathrm{~m}$ \\
\hline$A_{r c}$ & Strength of interaction force of car & $1.41 \mathrm{~m} / \mathrm{s} 2$ \\
\hline$B_{r c x}$ & $\begin{array}{l}\text { Influence range of longitudinal } \\
\text { interaction force of car }\end{array}$ & $4.78 \mathrm{~m}$ \\
\hline$B_{r c y}$ & $\begin{array}{l}\text { Influence range of lateral } \\
\text { interaction force of car }\end{array}$ & $2.32 \mathrm{~m}$ \\
\hline
\end{tabular}

\section{VALIDATION OF MODEL}

Using the model, for validating the accuracy of the model, a simulation program is created to conduct the simulation of the field survey traffic flow, and then using the simulation results to compare with the field survey data to validate the model accuracy. According to the value of RMSE 以及 MAPE, the results as illustrated in Table 5 show the accuracy of model applied to simulate the real traffic flow is acceptable.

$$
\begin{aligned}
& \text { RMSE }=\sqrt{\frac{1}{N} \sum_{n=1}^{N}\left(V^{s i m}-V^{o b s}\right)^{2}} \\
& M A P E=\frac{1}{N} \sum_{i=1}^{N}\left|\left(V^{s i m}-V^{o b s}\right) / V^{o b s}\right| \times 100 \%
\end{aligned}
$$

where $N$ : simulation time steps, $V^{\text {sim }}$ : output of simulation, $V^{o b s}$ : field survey value.

TABLE V. THE VALIDATION RESULTS OF RMSE AND MAPE

\begin{tabular}{cc}
\hline speed(m/s) & $\begin{array}{c}\text { Diving direction } \\
\text { angle }\end{array}$ \\
2.78 & 4 \\
$33.8 \%$ & $3 \%$ \\
\hline
\end{tabular}

\section{CONCLUSION AND SUGGESTION}

Using the force field concept, the mobility force model is developed in this paper through a preliminary calibration and validation process. The model is expected to enhance the simulation ability to simulate the complex driving behavior of motorcycle mixed traffic in the future.

\section{REFERENCES}

[1] Tien-Pen Hsu; Guo-Yu Weng and Yu-Jui Lin , Conceptual structure of a novel car-following model upon gravitational field concept, 9th ITS World Congress, Vienna, Austria, 22/26 October 2012

[2] Helbing D, Molnar P (1995), Social force model of pedestrian dynamics, Physical Review E 5, pp. 4282-4286.

[3] Hoogendoorn , S.P. (2005), Microscopic Calibration and Validation of Pedestrian Models - Cross-Comparison of Models using Experimental Data, Pedestrian and Evacuation Dynamic 2005, pp. 253-265. 